\title{
Fully commutative elements of type $D$ and homogeneous representations of KLR-algebras
}

\author{
Gabriel Feinberg and Kyu-Hwan LeE*
}

\begin{abstract}
In this paper, we decompose the set of fully commutative elements into natural subsets when the Coxeter group is of type $D_{n}$, and study combinatorics of these subsets, revealing hidden structures. (We do not consider type $A_{n}$ first, since a similar decomposition for type $A_{n}$ is trivial.) As an application, we classify and enumerate the homogeneous representations of the Khovanov-LaudaRouquier algebras of type $D_{n}$.

AMS 2000 SUBJECT Classifications: Primary 16G99, 00K01; secondary $05 \mathrm{E} 10$.

KEYWORDS AND PHRASES: Fully commutative elements, KLR algebra, homogeneous representation.
\end{abstract}

\section{Introduction}

An element $w$ of a Coxeter group is said to be fully commutative if any reduced word for $w$ can be obtained from any other by interchanges of adjacent commuting generators. These elements were first introduced by Fan [Fan95, Fan96] and Graham [Gra95] in their study of the generalized Temperley-Lieb algebras, where they showed that the generalized Temperley-Lieb algebras have a linear basis indexed by the fully commutative elements. Soon after, these elements were extensively studied by Stembridge in a series of papers [Ste96, Ste97, Ste98]. He gave a classification of the Coxeter groups having a finite number of fully commutative elements, which was previously done by Graham and Fan in the simply-laced case, and enumerated fully commutative elements for each of the finite types. The set of fully commutative elements also has connections to Kazhdan-Lusztig cells [Fan97, FG99, GL01].

In this paper, we study fully commutative elements of the Coxeter groups of type $D_{n}$. We decompose the set of fully commutative elements into natural subsets, and study combinatorial properties of these subsets. More precisely, the subsets obtained from the decomposition of the set of fully commutative

*This work was partially supported by a grant from the Simons Foundation (\#318706). 
elements are called packets. The main result (Theorem 2.12) shows that each set in the $(n, k)$-packet has size equal to $C(n, k)$, the $(n, k)$-entry of Catalan's triangle, and implies the following identity (Corollary 2.14):

$$
\sum_{k=0}^{n} C(n, k)|\mathcal{P}(n, k)|=\frac{n+3}{2} C_{n}-1,
$$

where $C_{n}$ is the $n^{\text {th }}$ Catalan number and $|\mathcal{P}(n, k)|$ is the number of elements in the $(n, k)$-packet.

It is quite intriguing that the set of fully commutative elements of type $D_{n}$ has such a structure. A similar decomposition of the set of fully commutative elements of type $A_{n}$ would be trivial as there would be only one packet. Since the right-hand side of $(0.1)$ is equal to the dimension of the Temperley-Lieb algebra of type $D_{n}$, the identity (0.1) suggests that there might be a representation theoretic construction, in which $C(n, k)$ would correspond to the dimension of a representation and $|\mathcal{P}(n, k)|$ to its multiplicity.

As a related work, Lejczyk and Stroppel studied these fully commutative elements of type $D_{n}$ and the Temperley-Lieb algebra action in their paper [LS13], where they also gave a diagrammatical description of the parabolic Kazhdan-Lusztig polynomials.

Actually, this research was motivated by the connection of the fully commutative elements to the homogeneous representations of the KhovanovLauda-Rouquier (KLR) algebras (also known as quiver Hecke algebras). Introduced by Khovanov and Lauda [KL09] and independently by Rouquier [Rou08], the KLR algebras have been the focus of many recent studies. In particular, these algebras categorify the lower (or upper) half of a quantum group. In the paper [KR11], Kleshchev and Ram significantly reduce the problem of describing the irreducible representations of the KLR algebras to the study of cuspidal representations for finite types. In the process of constructing the cuspidal representations, Kleshchev and Ram defined a class of representations known as homogeneous representations [KR10], those that are concentrated in a single degree. Homogeneous representations include most of the cuspidal representations for finite types with a suitable choice of ordering on words. In particular, for type $D_{n}$, a natural choice of ordering makes all the cuspidal representations homogeneous. Therefore it is important to completely understand homogeneous representations. The main point in our approach is that homogeneous representations can be constructed from the sets of reduced words of fully commutative elements in the corresponding Coxeter group as shown in [KR10]. 
As an application intended at the beginning of this research, our results classify and enumerate the homogeneous representations of KLR algebras according to the decomposition of the set of fully commutative elements. See Corollary 2.13.

The outline of this paper is as follows. In Section 1, we fix notations, briefly review the representations of KLR algebras, and explain the relationship between homogeneous representations and fully commutative elements of a Coxeter group. In the next section, we study the set of fully commutative elements of type $D_{n}$, construct explicit bijections among packets, prove the main theorem (Theorem 2.12) and obtain the identity (0.1).

\section{Acknowledgments}

The authors would like to thank Catharina Stroppel for helpful comments and the referee for many useful comments. Part of this research was performed while both authors were visiting the Institute for Computational and Experimental Research in Mathematics (ICERM) during the spring of 2013 for the special program "Automorphic Forms, Combinatorial Representation Theory and Multiple Dirichlet Series". They wish to thank the organizers and staff.

\section{Homogeneous representations and fully commutative elements}

\subsection{Definitions}

To define a KLR algebra, we begin with a quiver $\Gamma$. In this paper, we will focus mainly on quivers of Dynkin type $D_{n}$, but for the definition, any finite quiver with no double bonds will suffice. Let $I$ be the set indexing the vertices of $\Gamma$, and for indices $i \neq j$, we will say that $i$ and $j$ are neighbors if $i \rightarrow j$ or $i \leftarrow j$. Define $Q_{+}=\bigoplus_{i \in I} \mathbb{Z}_{\geq 0} \alpha_{i}$ as the non-negative lattice with basis $\left\{\alpha_{i} \mid i \in I\right\}$. The set of all words in the alphabet $I$ is denoted by $\langle I\rangle$, and for a fixed $\alpha=\sum_{i \in I} c_{i} \alpha_{i} \in Q_{+}$, let $\langle I\rangle_{\alpha}$ be the set of words $\boldsymbol{w}$ on the alphabet $I$ such that each $i \in I$ occurs exactly $c_{i}$ times in $\boldsymbol{w}$. We define the height of $\alpha$ to be $\sum_{i \in I} c_{i}$. We will write $\boldsymbol{w}=\left[w_{1}, w_{2}, \ldots, w_{d}\right], w_{j} \in I$.

Now, fix an arbitrary ground field $\mathbb{F}$ and choose an element $\alpha \in Q_{+}$. Then the Khovanov-Lauda-Rouquier algebra $R_{\alpha}$ is the associative $\mathbb{F}$-algebra generated by:

- idempotents $\left\{e(\boldsymbol{w}) \mid \boldsymbol{w} \in\langle I\rangle_{\alpha}\right\}$, 
- symmetric generators $\left\{\psi_{1}, \ldots, \psi_{d-1}\right\}$ where $d$ is the height of the root $\alpha$,

- polynomial generators $\left\{y_{1}, \ldots, y_{d}\right\}$,

subject to relations

$$
\begin{aligned}
& e(\boldsymbol{w}) e(\boldsymbol{v})=\delta_{\boldsymbol{w} \boldsymbol{v}} e(\boldsymbol{w}), \quad \sum_{\boldsymbol{w} \in\langle I\rangle_{\alpha}} e(\boldsymbol{w})=1 ; \\
& y_{k} e(\boldsymbol{w})=e(\boldsymbol{w}) y_{k} ; \\
& \psi_{k} e(\boldsymbol{w})=e\left(s_{k} \boldsymbol{w}\right) \psi_{k} ; \\
& y_{k} y_{\ell}=y_{\ell} y_{k} \text {; } \\
& y_{k} \psi_{\ell}=\psi_{\ell} y_{k}(\text { for } k \neq \ell, \ell+1) \text {; } \\
& \left(y_{k+1} \psi_{k}-\psi_{k} y_{k}\right) e(\boldsymbol{w})= \begin{cases}e(\boldsymbol{w}) & \text { if } w_{k}=w_{k+1}, \\
0 & \text { otherwise }\end{cases} \\
& \left(\psi_{k} y_{k+1}-y_{k} \psi_{k}\right) e(\boldsymbol{w})= \begin{cases}e(\boldsymbol{w}) & \text { if } w_{k}=w_{k+1} \\
0 & \text { otherwise }\end{cases} \\
& \psi_{k}^{2} e(\boldsymbol{w})= \begin{cases}0 & \text { if } w_{k}=w_{k+1}, \\
\left(y_{k}-y_{k+1}\right) e(\boldsymbol{w}) & \text { if } w_{k} \rightarrow w_{k+1}, \\
\left(y_{k+1}-y_{k}\right) e(\boldsymbol{w}) & \text { if } w_{k} \leftarrow w_{k+1}, \\
e(\boldsymbol{w}) & \text { otherwise; }\end{cases} \\
& \psi_{k} \psi_{\ell}=\psi_{\ell} \psi_{k}(\text { for }|k-\ell|>1) \text {; } \\
& \left(\psi_{k+1} \psi_{k} \psi_{k+1}-\psi_{k} \psi_{k+1} \psi_{k}\right) e(\boldsymbol{w}) \\
& = \begin{cases}e(\boldsymbol{w}) & \text { if } w_{k+2}=w_{k} \rightarrow w_{k+1}, \\
-e(\boldsymbol{w}) & \text { if } w_{k+2}=w_{k} \leftarrow w_{k+1}, \\
0 & \text { otherwise. }\end{cases}
\end{aligned}
$$

Here $\delta_{\boldsymbol{w} \boldsymbol{v}}$ in (1.1) is the Kronecker delta and, in (1.3), $s_{k}$ is the $k^{\text {th }}$ simple transposition in the symmetric group $S_{d}$, acting on the word $\boldsymbol{w}$ by swapping the letters in the $k^{\text {th }}$ and $(k+1)^{\text {st }}$ positions. If $\Gamma$ is a Dynkin-type quiver, we will say that $R_{\alpha}$ is a KLR algebra of that type.

We impose a $\mathbb{Z}$-grading on $R_{\alpha}$ by

$$
\begin{aligned}
& \operatorname{deg}(e(\boldsymbol{w}))=0, \quad \operatorname{deg}\left(y_{i}\right)=2, \\
& \operatorname{deg}\left(\psi_{i} e(\boldsymbol{w})\right)=\left\{\begin{aligned}
-2 & \text { if } w_{i}=w_{i+1}, \\
1 & \text { if } w_{i}, w_{i+1} \text { are neighbors in } \Gamma, \\
0 & \text { if } w_{i}, w_{i+1} \text { are not neighbors in } \Gamma .
\end{aligned}\right.
\end{aligned}
$$

Set $R=\bigoplus_{\alpha \in Q_{+}} R_{\alpha}$, and let $\operatorname{Rep}(R)$ be the category of finite dimensional 
graded $R$-modules, and denote its Grothendieck group by $[\operatorname{Rep}(R)]$. Then $\operatorname{Rep}(R)$ categorifies one half of the quantum group. More precisely, let $\mathbf{f}$ and 'f be the Lusztig's algebras defined in [Lus11, Section 1.2] attached to the Cartan datum encoded in the quiver $\Gamma$ over the field $\mathbb{Q}(v)$. We put $q=v^{-1}$ and $\mathcal{A}=\mathbb{Z}\left[q, q^{-1}\right]$, and let ' $\mathbf{f}_{\mathcal{A}}$ and $\mathbf{f}_{\mathcal{A}}$ be the $\mathcal{A}$-forms of ' $\mathbf{f}$ and $\mathbf{f}$, respectively. Consider the graded duals ' $\mathbf{f}^{*}$ and $\mathbf{f}^{*}$, and their $\mathcal{A}$-forms

$$
{ }^{\prime} \mathbf{f}_{\mathcal{A}}^{*}:=\left\{x \in{ }^{\prime} \mathbf{f}^{*}: x\left({ }^{\prime} \mathbf{f}_{\mathcal{A}}\right) \subset \mathcal{A}\right\} \text { and } \mathbf{f}_{\mathcal{A}}^{*}:=\left\{x \in \mathbf{f}^{*}: x\left(\mathbf{f}_{\mathcal{A}}\right) \subset \mathcal{A}\right\} .
$$

Then we have:

Theorem 1.1. [KL09] There is an A-linear (bialgebra) isomorphism $\gamma^{*}$ : $[\operatorname{Rep}(R)] \stackrel{\sim}{\rightarrow} \mathbf{f}_{\mathcal{A}}^{*}$.

Since it is not directly related to our purpose, we omit defining the bialgebra structures. Details can be found in [KL09, KR11].

A word $\mathbf{i} \in\langle I\rangle_{\alpha}$ is naturally considered as an element of ' $\mathbf{f}_{\mathcal{A}}^{*}$ to be dual to the corresponding monomial in ' $\mathbf{f}_{\mathcal{A}}$. Let $M$ be a finite dimensional graded $R_{\alpha}$-module. Define the $q$-character of $M$ by

$$
\operatorname{ch}_{q} M:=\sum_{\mathbf{i} \in\langle I\rangle_{\alpha}}\left(\operatorname{dim}_{q} M_{\mathbf{i}}\right) \mathbf{i} \in \mathbf{f}_{\mathcal{A}}^{*},
$$

where $M_{\mathbf{i}}=e(\mathbf{i}) M$ and $\operatorname{dim}_{q} V:=\sum_{n \in \mathbb{Z}}\left(\operatorname{dim} V_{n}\right) q^{n} \in \mathcal{A}$ for $V=\oplus_{n \in \mathbb{Z}} V_{n}$. A non-empty word $\mathbf{i}$ is called Lyndon if it is lexicographically smaller than all its proper right factors, or equivalently smaller that all its rotations. For $x \in{ }^{\prime} \mathbf{f}^{*}$ we denote by $\max (x)$ the largest word appearing in $x$. A word $\mathbf{i} \in\langle I\rangle$ is called good if there is $x \in \mathbf{f}^{*}$ such that $\mathbf{i}=\max (x)$. Given a module $L \in \operatorname{Rep}\left(R_{\alpha}\right)$, we say that $\mathbf{i} \in\langle I\rangle$ is the highest weight of $L$ if $\mathbf{i}=\max \left(\operatorname{ch}_{q} L\right)$. An irreducible module $L \in R_{\beta}$ is called cuspidal if its highest weight is a good Lyndon word. It is known that the set of good Lyndon words is in bijection with the set of positive roots $\Delta_{+}$of the root system attached to $\Gamma$, when $\Gamma$ is of finite type.

Using the techniques developed by Leclerc in [Lec04], Kleshchev and Ram, and then Melvin, Mondragon, and Hill showed:

Theorem 1.2 ([KR11]; [HMM12], 4.1.1). Assume that $\Gamma$ is of finite Dynkin type. Then the good Lyndon words parameterize the cuspidal representations of the KLR algebra $R_{\beta}, \beta \in \Delta_{+}$. In turn, any irreducible graded $R_{\alpha}$-module for $\alpha \in Q_{+}$is given by an irreducible head of a standard representation induced from cuspidal representations up to isomorphism and degree shift. 
The above theorem clearly explains the importance of cuspidal representations. In the next subsection, we will introduce another class of representations which contains most of the cuspidal representations with a suitable choice of ordering on words.

\subsection{Homogeneous representations}

We define a homogeneous representation of a KLR algebra to be an irreducible, graded representation fixed in a single degree (with respect to the $\mathbb{Z}$-grading described in (1.11) and (1.12)). Homogeneous representations form an important class of irreducible modules since most of the cuspidal representations are homogeneous with a suitable choice of ordering on $\langle I\rangle$ ([KR11, HMM12]). After introducing some terminology, we will describe these representations in a combinatorial way. We continue to assume that $\Gamma$ is a simply-laced quiver.

Fix an $\alpha \in Q_{+}$and let $d$ be the height of $\alpha$. For any word $\boldsymbol{w} \in\langle I\rangle_{\alpha}$, we say that the simple transposition $s_{r} \in S_{d}$ is an admissible transposition for $\boldsymbol{w}$ if the letters $w_{r}$ and $w_{r+1}$ are neither equal nor neighbors in the quiver $\Gamma$. Following Kleshchev and Ram [KR10], we define the weight graph $G_{\alpha}$ with vertices given by $\langle I\rangle_{\alpha}$. Two words $\boldsymbol{w}, \boldsymbol{v} \in\langle I\rangle_{\alpha}$ are connected by an edge if there is an admissible transposition $s_{r}$ such that $s_{r} \boldsymbol{w}=\boldsymbol{v}$.

We say that a connected component $C$ of the weight graph $G_{\alpha}$ is homogeneous if the following property holds for every $\boldsymbol{w} \in C$ :

If $w_{r}=w_{s}$ for some $1 \leq r<s \leq d$, then there exist $t, u$

with $r<t<u<s$ such that $w_{r}$ is neighbors with both $w_{t}$ and $w_{u}$.

A word satisfying condition (1.13) will be called a homogeneous word.

Example 1.3. Consider the $A_{3}$ quiver

$$
\Gamma=\underset{1}{\bullet \longrightarrow} \underset{2}{\longrightarrow} \stackrel{\bullet}{\leftarrow}
$$

We then have $I=\{1,2,3\}$, and choose the element $\alpha=\alpha_{1}+2 \alpha_{2}+\alpha_{3} \in Q_{+}$. Then the weight graph $G_{\alpha}$ is given by:

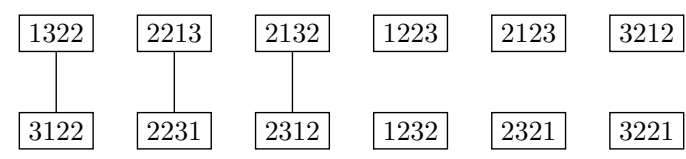


One can see that the only homogeneous component is

$$
\begin{array}{|l|}
\hline 2132 \\
\hline \multicolumn{2}{|r|}{} \\
\hline 2312 \\
\hline
\end{array}
$$

In this case, the two instances of the letter 2 have the neighbors 1 and 3 occurring between them. Note that, for some $\alpha \in Q_{+}$we may have that every component of the weight graph is homogeneous (e.g. $\alpha=\alpha_{1}+\alpha_{2}+\alpha_{3}$ ), while for others we may see that no components are homogeneous (e.g. $\left.\alpha=2 \alpha_{1}+\alpha_{2}\right)$.

A main theorem of [KR10] shows that the homogeneous components of $G_{\alpha}$ exactly parameterize the homogeneous representations of the KLR algebra $R_{\alpha}$ :

Theorem 1.4 ([KR10], Theorem 3.4). Let $C$ be a homogeneous component of the weight graph $G_{\alpha}$. Define an $\mathbb{F}$-vector space $S(C)$ with basis $\left\{v_{\boldsymbol{w}} \mid \boldsymbol{w} \in\right.$ $C$ ) labeled by the vertices in $C$. Then we have an $R_{\alpha}$-action on $S(C)$ given by

$$
\begin{aligned}
e\left(\boldsymbol{w}^{\prime}\right) v_{\boldsymbol{w}} & =\delta_{\boldsymbol{w}, \boldsymbol{w}^{\prime}} v_{\boldsymbol{w}} \quad\left(\boldsymbol{w}^{\prime} \in\langle I\rangle_{\alpha}, \boldsymbol{w} \in C\right), \\
y_{r} v_{\boldsymbol{w}} & =0 \quad(1 \leq r \leq d, \boldsymbol{w} \in C), \\
\psi_{r} v_{\boldsymbol{w}} & =\left\{\begin{array}{ll}
v_{s_{r} \boldsymbol{w}} & \text { if } s_{r} \boldsymbol{w} \in C \\
0 & \text { otherwise }
\end{array} \quad(1 \leq r \leq d-1, \boldsymbol{w} \in C),\right.
\end{aligned}
$$

which gives $S(C)$ the structure of a homogeneous, irreducible $R_{\alpha}$-module. Further $S(C) \nsubseteq S\left(C^{\prime}\right)$ if $C \neq C^{\prime}$, and this construction gives all of the irreducible homogeneous modules, up to isomorphism.

As a result, the task of identifying homogeneous modules of a KLR algebra is reduced to identifying homogeneous components in a weight graph. This is simplified further by the following lemma:

Lemma 1.5 ([KR10], Lemma 3.3). A connected component $C$ of the weight graph $G_{\alpha}$ is homogeneous if and only if an element $\boldsymbol{w} \in C$ satisfies the condition (1.13).

Recall that we call a word satisfying condition (1.13) a homogeneous word. The homogeneous words have other combinatorial characterizations, which we explore in the next subsection. 


\subsection{Fully commutative elements of Coxeter groups}

Since the homogeneity of $\boldsymbol{w} \in\langle I\rangle$ does not depend on the orientation of a quiver, it is enough to consider Dynkin diagrams and the corresponding Coxeter groups. Given a simply-laced Dynkin diagram, the corresponding Coxeter group will be denoted by $W$ and the generators by $s_{i}, i \in I$. A reduced expression $s_{i_{1}} \cdots s_{i_{r}}$ will be identified with the word $\left[i_{1}, \ldots, i_{r}\right]$ in $\langle I\rangle$. For example, in type $A_{4}$, the reduced expressions

$$
s_{3} s_{1} s_{2} s_{3} s_{4}=s_{1} s_{3} s_{2} s_{3} s_{4}=s_{1} s_{2} s_{3} s_{2} s_{4}=s_{1} s_{2} s_{3} s_{4} s_{2}
$$

are identified with the words

$[3,1,2,3,4],[1,3,2,3,4],[1,2,3,2,4],[1,2,3,4,2], \quad$ respectively.

The identity element will be identified with the empty word [ ].

Assume that $W$ is a simply-laced Coxeter group. An element $w \in W$ is said to be fully commutative if any reduced word for $w$ can be obtained from any other by interchanges of adjacent commuting generators, or equivalently if no reduced word for $w$ has $\left[i, i^{\prime}, i\right]$ as a subword where $i$ and $i^{\prime}$ are neighbors in the Dynkin diagram. We make several observations, which are important for our study of homogeneous representations, and list them in the following lemma. These observations were first made by Kleshchev and Ram.

Lemma 1.6. [KR10]

(1) A homogeneous component of the weight graph $G_{\alpha}$ contains as its vertices exactly the set of reduced expressions for a fully commutative element in $W$.

(2) The set of homogeneous components is in bijection with the set of fully commutative elements in $W$.

(3) Any KLR algebra of type $A_{n}(n \geq 1), D_{n}(n \geq 4)$, or $E_{n}(n=6,7,8)$ has finitely many irreducible homogeneous representations.

Proof. Part (1) follows from the definitions; in particular, the condition (1.13) implies that no word in a homogeneous component has $\left[i, i^{\prime}, i\right]$ as a subword where $i$ and $i^{\prime}$ are neighbors. Parts (2) and (3) are consequences of (1).

Stembridge [Ste96] classified all of the Coxeter groups that have finitely many fully commutative elements. The list includes the infinite families of types $E_{n}, F_{n}$ and $H_{n}$. His results completed the work of Fan [Fan96], who 
had done this for the simply-laced types. In the same paper [Fan96], Fan showed that the fully commutative elements parameterized natural bases for corresponding quotients of Hecke algebras. In type $A_{n}$, these give rise to the Temperley-Lieb algebras (see [Jon87]). Fan and Stembridge also enumerated the set of fully commutative elements. In particular, they showed the following.

Proposition 1.7 ([Fan96, Ste98]). Let $C_{n}$ be the $n^{\text {th }}$ Catalan number, i.e. $C_{n}=\frac{1}{n+1}\left(\begin{array}{c}2 n \\ n\end{array}\right)$. Then the number of fully commutative elements in the Coxeter group of type $A_{n}$ is $C_{n+1}$, and that of type $D_{n}$ is $\frac{n+3}{2} C_{n}-1$.

We immediately obtain a consequence on homogeneous representations from Lemma 1.6.

Corollary 1.8. A KLR algebra $R=\bigoplus_{\alpha \in Q_{+}} R_{\alpha}$ of type $A_{n}$ has $C_{n+1}$ irreducible homogeneous representations, while a KLR algebra of type $D_{n}$ has $\frac{n+3}{2} C_{n}-1$ irreducible homogeneous representations.

In [KR10], Kleshchev and Ram parameterized homogeneous representations using skew shapes. In this paper, we will decompose the set of fully commutative elements to give a finer enumeration of homogeneous representations in type $D_{n}$. More precisely, our main theorem (Theorem 2.12) proves that these homogeneous representations can be organized naturally into packets (defined in Section 2.2), and counted by Catalan's triangle. Note that these results contribute not only to the combinatorics of the representation theory of KLR algebras, but also to the study of fully commutative elements of Coxeter groups.

\section{Packets in type $D_{n}$}

In this section, we will focus on fully commutative elements and representations of KLR-algebras of type $D_{n}$. That is, we shall assume that $\Gamma$ is a quiver whose underlying graph is of the form:

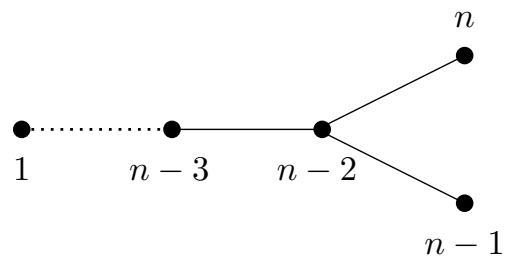

We begin with canonical reduced words of type $D_{n}$. 


\subsection{Canonical reduced words}

For $1 \leq i \leq n-1$, we define the words $s_{i j}$ by:

$$
s_{i j}= \begin{cases}{[i, i-1, \ldots, j]} & \text { if } i>j \\ {[i]} & \text { if } i=j \\ {[]} & \text { if } i<j\end{cases}
$$

When $i=n$, we define

$$
s_{n j}= \begin{cases}{[n, n-2, \ldots, j]} & \text { if } j \leq n-2, \\ {[n]} & \text { if } j=n, n-1 \\ {[]} & \text { if } j>n\end{cases}
$$

We will often write $s_{i i}=s_{i}$. The following lemma provides a canonical form we need.

Lemma 2.1 ([BS01], Lemma 5.2). Any element of the Coxeter group of type $D_{n}$ can be uniquely written in the reduced form

$$
s_{1 i_{1}} s_{2 i_{2}} \cdots s_{n-1 i_{n-1}} s_{n j_{1}} s_{n-1 j_{2}} s_{n j_{3}} s_{n-1 j_{4}} \cdots s_{n-1+[\ell]_{2} j_{\ell}}
$$

where $1 \leq i_{k} \leq k+1$ for $1 \leq k \leq n-1$, and $1 \leq j_{1}<j_{2}<\cdots<j_{\ell} \leq n-1$ for $\ell \geq 0$, and $[\ell]_{2}=1$ when $\ell$ is odd, $[\ell]_{2}=0$ when $\ell$ is even.

The left factor $s_{1 i_{1}} s_{2 i_{2}} \cdots s_{n-1 i_{n-1}}$ will be called the prefix, and similarly the right factor $s_{n j_{1}} s_{n-1 j_{2}} s_{n j_{3}} s_{n-1 j_{4}} \cdots s_{n-1+[\ell]_{2} j_{\ell}}$ will be called the suffix of the reduced word. For example, in the case of $D_{5}$, the word $s_{21} s_{3} s_{4} s_{52} s_{43} s_{5}=$ $[2,1,3,4,5,3,2,4,3,5]$ has prefix $s_{21} s_{3} s_{4}=[2,1,3,4]$ and suffix $s_{52} s_{43} s_{5}=$ $[5,3,2,4,3,5]$. Given a reduced word $\boldsymbol{w}$ in canonical form, we will denote by $\boldsymbol{w}_{0}$ the prefix of $\boldsymbol{w}$ and by $\boldsymbol{w}^{\prime}$ the suffix, and write $\boldsymbol{w}=\boldsymbol{w}_{0} \boldsymbol{w}^{\prime}$. Generally, a word of the form $s_{n j_{1}} s_{n-1 j_{2}} s_{n j_{3}} s_{n-1 j_{4}} \cdots s_{n-1+[\ell]_{2} j_{\ell}}$ with $1 \leq j_{1}<j_{2}<$ $\cdots<j_{\ell} \leq n-1$ for $\ell \geq 0$ will be called a suffix.

Remark 2.2. Notice that choosing a suffix is equivalent to choosing a (possibly empty) subset of $\{1,2, \ldots, n-1\}$. There are $2^{n-1}$ ways to do this. Since there are $n$ ! prefixes, we have $n ! \cdot 2^{n-1}$ elements in the canonical reduced form. We recall that there are the same number of elements in the type $D_{n}$ Coxeter group.

Lemma 2.3. Every suffix is a homogeneous word, and so represents a fully commutative element. 
Proof. A suffix $\boldsymbol{w}^{\prime}$ cannot have $[1, \ldots, 1]$ as a subword. If a suffix $\boldsymbol{w}^{\prime}$ has a subword $[k, \ldots, k]$ for $2 \leq k \leq n-2$, then $\boldsymbol{w}^{\prime}$ must have $[k, k-1, \ldots, k+1, k]$, or possibly $[n-2, n-3, \ldots, n, n-2]$ if $k=n-2$, as a subword from the conditions on a suffix. In either case, $\boldsymbol{w}^{\prime}$ is a homogeneous word from the definition (1.13). If $\boldsymbol{w}^{\prime}$ has $[n-1, \ldots, n-1]$ as a subword, then $\boldsymbol{w}^{\prime}$ must have $[n-1, n-2, \ldots, n, n-2, \ldots, n-1]$. Similarly, if $\boldsymbol{w}^{\prime}$ has $[n, \ldots, n]$, then $\boldsymbol{w}^{\prime}$ must have $[n, n-2, \ldots, n-1, n-2, \ldots, n]$. In both cases, $\boldsymbol{w}^{\prime}$ is a homogeneous word.

Let $\mathcal{W}_{n}$ be the set of canonical reduced words of type $D_{n}$ given in Lemma 2.1. Each homogeneous word in $\mathcal{W}_{n}$ uniquely represents a fully commutative element of the Coxeter group of type $D_{n}$ and also a homogeneous component of a weight graph by Lemma 1.6. The homogeneous words in $\mathcal{W}_{n}$ will be grouped based on their suffixes:

Definition 2.4. A collection $\mathbf{c}_{\boldsymbol{w}^{\prime}}^{n} \subset \mathcal{W}_{n}$ labeled by a suffix $\boldsymbol{w}^{\prime}$ is defined to be the set of homogeneous words in $\mathcal{W}_{n}$ whose suffix is $\boldsymbol{w}^{\prime}$. A collection will be identified with the set of corresponding fully commutative elements in the Coxeter group of type $D_{n}$.

Some of the collections have the same number of elements as we will see in the following lemma and proposition.

Lemma 2.5. For a fixed $k, 0 \leq k \leq n-3$, any collection labeled by a suffix of the form

$$
s_{n k+1} s_{n-1 j_{2}} s_{n j_{3}} s_{n-1 j_{4}} \cdots s_{n-1+[\ell]_{2} j_{\ell}} \quad(\ell \geq 2)
$$

has the same set of prefixes. In particular, these collections have the same number of elements.

Proof. Let $\boldsymbol{w}^{\prime}$ be a suffix of the form (2.1). Then $\boldsymbol{w}^{\prime}$ has the suffix $\boldsymbol{w}_{1}:=$ $s_{n k+1} s_{n-1}$ as a subword. Since removing letters from the end of a word will not affect its homogeneity, it is clear that any prefix appearing in the collection $\mathbf{c}_{\boldsymbol{w}^{\prime}}^{n}$ also appears in $\mathbf{c}_{\boldsymbol{w}_{1}}^{n}$. We need to show, then, the opposite inclusion.

Suppose now that $\boldsymbol{w}_{0}$ is a prefix of a homogeneous word appearing in the collection labeled by $\boldsymbol{w}_{1}$. Since the prefix and suffix of a homogeneous word are individually homogeneous words, we only assume that there is some letter $r$ which appears in both $\boldsymbol{w}_{0}$ and $\boldsymbol{w}^{\prime}$. From the condition

$$
1 \leq j_{1}<j_{2}<\cdots<j_{\ell} \leq n-1
$$


on the suffix $\boldsymbol{w}^{\prime}$, we see that the letter $r$ also appears in $\boldsymbol{w}_{1}$. The homogeneity of $\boldsymbol{w}_{0} \boldsymbol{w}_{1}$ requires that two neighbors of $r$ appear between the instance of $r$ in $\boldsymbol{w}_{0}$ and the instance of $r$ in $\boldsymbol{w}_{1}$, and these two neighbors of $r$ also satisfy the homogeneity condition for $\boldsymbol{w}_{0} \boldsymbol{w}^{\prime}$ since $\boldsymbol{w}_{1}$ is a left factor of $\boldsymbol{w}^{\prime}$. This proves that $\boldsymbol{w}_{0}$ is a prefix of $\boldsymbol{w}^{\prime}$ for any suffix $\boldsymbol{w}^{\prime}$ of the form (2.1).

Proposition 2.6. For $1 \leq k \leq n-3$, the collection labeled by the suffix $s_{n k}$ has the same number of elements as any of the collections labeled by the suffix of the form

$$
s_{n k+1} s_{n-1 j_{2}} s_{n j_{3}} s_{n-1 j_{4}} \cdots s_{n-1+[\ell]_{2} j_{\ell}} \quad(\ell \geq 2)
$$

Proof. Let $\boldsymbol{w}_{1}=s_{n k+1} s_{n-1}$ and $\boldsymbol{w}_{2}=s_{n k}$. By Lemma 2.5, it is enough to establish a bijection between the collections $\mathbf{c}_{\boldsymbol{w}_{1}}^{n}$ and $\mathbf{c}_{\boldsymbol{w}_{2}}^{n}$. We define a map $\sigma: \mathbf{c}_{\boldsymbol{w}_{2}}^{n} \rightarrow \mathbf{c}_{\boldsymbol{w}_{1}}^{n}$ as follows. Suppose that $\boldsymbol{w}_{0}$ is the prefix of the word $\boldsymbol{w}=\boldsymbol{w}_{0} \boldsymbol{w}_{2}=\boldsymbol{w}_{0}[n, n-2, \ldots, k] \in \mathbf{c}_{\boldsymbol{w}_{2}}^{n}$, and let $r$ be the last letter of $\boldsymbol{w}_{0}$. Then by the condition of homogeneity, we must have $r<k$ or $r=n-1$.

If $r<k$, we define $\sigma(\boldsymbol{w})=\boldsymbol{w}_{0} \boldsymbol{w}_{1}$, i.e. the map $\sigma$ will simply replace the suffix $\boldsymbol{w}_{2}=[n, n-2, \ldots, k+1, k]$ with the suffix $\boldsymbol{w}_{1}=[n, n-2, \ldots, k+$ $1, n-1]$. To see that this image is actually in $\mathbf{c}_{\boldsymbol{w}_{1}}^{n}$, we need to check that changing the last letter of the suffix from $k$ to $n-1$ does not violate homogeneity. In turn, we need only to consider the case when $\sigma(\boldsymbol{w})$ has $\left[n-1, n-2, \ldots, i_{n-1}, n, n-2, \ldots, k+1, n-1\right]$ as a right factor. Clearly the neighbor $n-2$ appears twice between the two occurrences of $n-1$ and $\sigma(\boldsymbol{w}) \in \mathbf{c}_{\boldsymbol{w}_{1}}^{n}$ in this case.

If $r=n-1$, we take $m \geq k$ to be the smallest letter such that the string $[m, m+1, \ldots, n-1]$ is a right factor of $\boldsymbol{w}_{0}$. Then we have $\boldsymbol{w}=$ $s_{1 i_{1}} \cdots s_{m-1 i_{m-1}} s_{m} s_{m+1} \cdots s_{n-1} \boldsymbol{w}_{2}$, and we define

$$
\sigma(\boldsymbol{w})=s_{1 i_{1}} \cdots s_{m-1 i_{m-1}} s_{m k} \boldsymbol{w}_{1}
$$

In other words, the map $\sigma$ replaces the factor $s_{m} s_{m+1} \cdots s_{n-1}=[m, m+$ $1, \ldots, n-1]$ with the factor $s_{m k}=[m, m-1, \ldots, k]$ in addition to changing the suffix from $\boldsymbol{w}_{2}$ to $\boldsymbol{w}_{1}$.

We show now that the image $\sigma(\boldsymbol{w})=s_{1 i_{1}} \cdots s_{m-1 i_{m-1}} s_{m k} \boldsymbol{w}_{1}$ is still a homogeneous word. It is not hard to check that the right factor $s_{m k} \boldsymbol{w}_{1}$ is homogeneous, but suppose that some letter $t$ appears in both the segment $s_{m k}$ and in some segment $s_{j i_{j}}, j \leq m-1$. Then we have $k \leq t \leq j \leq$ $m-1 \leq n-2$. Since $t<m$, the letter $t$ does not appear in the ascending string $[m, \ldots, n-1]$ in the word $\boldsymbol{w}$, but since $k \leq t \leq n-2$, it does appear in the suffix, $[n, \ldots, t+1, t, \ldots, k]$, along with one neighbor $t+1$. Since $\boldsymbol{w}$ 
is homogeneous, there must have been another neighbor in the prefix $\boldsymbol{w}_{0}$ which is not touched by the action of $\sigma$. Thus $\sigma(\boldsymbol{w})$ is also a homogeneous word, and we have shown that $\sigma\left(\mathbf{c}_{\boldsymbol{w}_{2}}^{n}\right) \subset \mathbf{c}_{\boldsymbol{w}_{1}}^{n}$.

To summarize, we have

$\sigma(\boldsymbol{w})=\left\{\begin{aligned} s_{1 i_{1}} \cdots s_{m-1 i_{m-1}} s_{m k} \boldsymbol{w}_{1} & \text { if } \boldsymbol{w}_{0} \text { ends with }[m, m+1, \ldots, n-1], \\ \boldsymbol{w}_{0} \boldsymbol{w}_{1} & \text { otherwise, }\end{aligned}\right.$

where $m \geq k$. Note that $\boldsymbol{w}_{0}$ can not end in $n-1$ in the second case. For example, when $n=5$ and $k=2$, we have

\begin{tabular}{|c|c|}
\hline $\boldsymbol{w} \in \mathbf{c}_{[5,3,2]}^{5}$ & $\sigma(\boldsymbol{w}) \in \mathbf{c}_{[5,3,4]}^{5}$ \\
\hline \hline$[3,2,1,5,3,2]$ & {$[3,2,1,5,3,4]$} \\
\hline$[4,3,2,1,5,3,2]$ & {$[4,3,2,1,5,3,4]$} \\
\hline$[1,2,3,4,5,3,2]$ & {$[1,2,5,3,4]$} \\
\hline$[2,1,4,5,3,2]$ & {$[2,1,4,3,2,5,3,4]$} \\
\hline
\end{tabular}

Next, we define a map that goes in the other direction, $\tau: \mathbf{c}_{\boldsymbol{w}_{1}}^{n} \rightarrow \mathbf{c}_{\boldsymbol{w}_{2}}^{n}$. Suppose that $\boldsymbol{w}=\boldsymbol{w}_{0} \boldsymbol{w}_{1}=\boldsymbol{w}_{0}[n, n-2, \ldots, k+1, n-1] \in \mathbf{c}_{\boldsymbol{w}_{1}}^{n}$, and let $r$ be the last letter of $\boldsymbol{w}_{0}$. Then by the condition of homogeneity, we must have $1 \leq r \leq k$.

If $r<k$, then we define $\tau\left(\boldsymbol{w}_{0} \boldsymbol{w}_{1}\right)=\boldsymbol{w}_{0} \boldsymbol{w}_{2}$, i.e. $\tau$ simply replaces the suffix $[n, \ldots, k+1, n-1]$ with the suffix $[n, \ldots, k+1, k]$. To see that this results in a homogeneous word, we need to check only that the letter $k$ at the end of the suffix does not violate the homogeneity condition. Assume that another $k$ appears in $\boldsymbol{w}_{0}$, and consider the last non-empty segment $s_{m r}$ of $\boldsymbol{w}_{0}$. Then we have $r<k \leq m$. Since there are two neighbors $(k+1$ and $k-1$ ) between the two occurrences of $k$, homogeneity is preserved.

If $r=k$, then the final non-empty segment of the prefix is $s_{m k}$ for some $m$ with $k \leq m \leq n-1$. We define

$$
\tau(\boldsymbol{w})=\tau\left(s_{1 i_{1}} \cdots s_{m-1 i_{m-1}} s_{m k} \boldsymbol{w}_{1}\right)=s_{1 i_{1}} \cdots s_{m-1 i_{m-1}} s_{m} s_{m+1} \cdots s_{n-1} \boldsymbol{w}_{2} .
$$

That is, $\tau$ replaces $s_{m k}$ with the ascending string $[m, m+1, \ldots, n-1]$ and the suffix $\boldsymbol{w}_{1}$ with $\boldsymbol{w}_{2}$. It remains to see that $\tau(\boldsymbol{w})$ is in fact a homogeneous word. Notice that the left factor $s_{1 i_{1}} \cdots s_{m-1 i_{m-1}}$ and the ascending string $[m, \ldots, n-1]$ have no letters in common, so there is nothing here to check. Also the right factor $[m, \ldots, n-1, n, n-2, \ldots, k]$ is easily checked to be homogeneous. Suppose that some letter $r$ occurs in the left factor $s_{1 i_{1}} \cdots s_{m-1 i_{m-1}}$, and also in the suffix $[n, n-2, \ldots, k]$. Since $r$ does not appear in the ascending string $[m, \ldots, n-1]$ but does appear in the suffix $[n, n-2, \ldots, k]$, it follows that $k \leq r<m$, so the letter $r$ appears in 
the segment $s_{m k}$ in $\boldsymbol{w}_{0}$. Since the word $\boldsymbol{w}_{0}$ is homogeneous, it must be the case that two neighbors of $r$ appear between these two instances of $r$ in $\boldsymbol{w}_{0}$. One of them may be in the segment $s_{m k}$ which is replaced by the map $\tau$, but at least one of them must be in the left factor which remains fixed under $\tau$. Clearly another neighbor occurs in the suffix, so the condition for homogeneity is satisfied.

To summarize, we have, for $\boldsymbol{w}=\boldsymbol{w}_{0} \boldsymbol{w}_{1} \in \mathbf{c}_{\boldsymbol{w}_{1}}^{n}$

$$
\tau(\boldsymbol{w})=\left\{\begin{aligned}
s_{1 i_{1}} \cdots s_{m-1 i_{m-1}} s_{m} s_{m+1} \cdots s_{n-1} \boldsymbol{w}_{2} & \text { if } \boldsymbol{w}_{0} \text { ends with } s_{m k}, \\
\boldsymbol{w}_{0} \boldsymbol{w}_{2} & \text { otherwise }
\end{aligned}\right.
$$

where $k \leq m \leq n-1$. Note that $\boldsymbol{w}_{0}$ can not end in $k$ in the second case. For example, when $n=5$ and $k=2$, we have

\begin{tabular}{|c|c|}
\hline $\boldsymbol{w} \in \mathbf{c}_{[5,3,4]}^{\mathbf{5}}$ & $\tau(\boldsymbol{w}) \in \mathbf{c}_{[5,3,2]}^{\mathbf{5}}$ \\
\hline \hline$[2,1,5,3,4]$ & {$[2,1,5,3,2]$} \\
\hline$[2,5,3,4]$ & {$[2,3,4,5,3,2]$} \\
\hline$[1,4,3,2,5,3,4]$ & {$[1,4,5,3,2]$} \\
\hline
\end{tabular}

Now one can check that $\tau$ is both a left and a right inverse of $\sigma$, so the bijection is established.

\subsection{Packets}

The results in the previous subsection show that some collections have the same cardinality. It is natural, then, to group them together, which lead us to the following definition.

Definition 2.7. For $0 \leq k \leq n$, we define the $(n, k)$-packet of collections:

- The $(n, 0)$-packet is the set of collections labeled by suffixes of the form

$$
s_{n 1} s_{n-1 j_{2}} s_{n j_{3}} s_{n-1 j_{4}} \cdots s_{n-1+[\ell]_{2} j_{\ell}} \quad(\ell \geq 2) .
$$

- The $(n, k)$-packet, $1 \leq k \leq n-3$, is the set of collections labeled by $s_{n k}$ or suffixes of the form $s_{n k+1} s_{n-1 j_{2}} s_{n j_{3}} s_{n-1 j_{4}} \cdots s_{n-1+[\ell]_{2} j_{\ell}} \quad(\ell \geq 2)$.

- The $(n, n-2)$-packet contains only the collection labeled by $s_{n n-2}=$ $[n, n-2]$.

- The $(n, n-1)$-packet contains only the collection labeled by $s_{n}=[n]$.

- The $(n, n)$-packet contains only the collection labeled by the empty suffix [ ]. 
We will denote the $(n, k)$-packet by $\mathcal{P}(n, k)$. As an example, Table 2.1 shows all of the packets in the case of $D_{4}$.

We record an important property of a packet:

Corollary 2.8. The collections in a packet have the same number of elements.

Proof. The assertion follows from Lemma 2.5 and Proposition 2.6.

We count the number of collections in a packet and obtain:

Proposition 2.9. The size of the packet $\mathcal{P}(n, k)$ is

$$
|\mathcal{P}(n, k)|=\left\{\begin{array}{cl}
2^{n-2}-1 & \text { if } k=0, \\
2^{n-k-2} & \text { if } 1 \leq k \leq n-2, \\
1 & \text { if } k=n-1, n .
\end{array}\right.
$$

It may be convenient to visualize these values in an array as in Table 2.2, where the row is given by $n$ (starting at 0 ), and the column is given by $k$ (also beginning at 0 ).

Proof. If $k=0$, a collection in the $(n, 0)$-packet is determined by a sequence $1<j_{2}<\cdots<j_{\ell} \leq n-1, \ell \geq 2$. The number of such sequences is the same as the number of non-empty subsets of $\{2,3, \ldots, n-1\}$, which is $2^{n-2}-1$. For $1 \leq k \leq n-3$, a collection in the $(n, k)$-packet is labeled by $s_{n k}$ or is determined by a sequence $k+1<j_{2}<\cdots<j_{\ell} \leq n-1, \ell \geq 2$. The number of such sequences is the same as the number of non-empty subsets of $\{k+2, k+3, \ldots, n-1\}$, which is $2^{n-k-2}-1$. Hence the total number of collections in the $(n, k)$-packet is $2^{n-k-2}$. The remaining cases $k=n-2, n-1, n$ are obvious.

Remark 2.10. Recall that there are $2^{n-1}$ suffixes in total, and as a check, we see that

$$
\sum_{k=0}^{n}|\mathcal{P}(n, k)|=2^{n-1} .
$$

\subsection{Catalan's triangle}

In this subsection, we will compute the size of a collection in a given packet, allowing us to classify and enumerate all homogeneous representations. We begin by presenting a seemingly unrelated sequence.

The array shown in Table 2.3 is known as Catalan's Triangle [OEI]. The entry in the $n^{\text {th }}$ row and $k^{\text {th }}$ column is denoted by $C(n, k)$, for $0 \leq k \leq n$. 


\section{$\mathcal{P}(4,0)$}

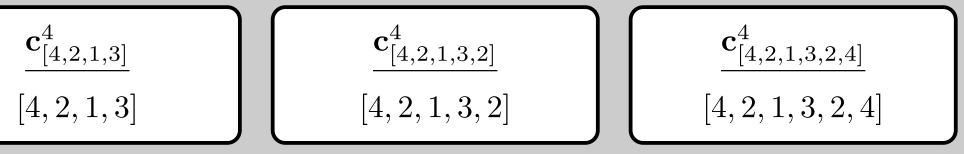

$\mathcal{P}(4,1)$

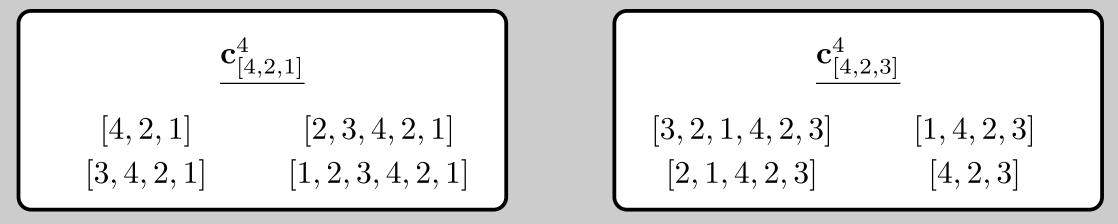

\section{$\mathcal{P}(4,2)$}

\begin{tabular}{ccc} 
& $\mathbf{c}_{[4,2]}^{4}$ & \\
{$[4,2]$} & {$[1,4,2]$} & {$[3,4,2]$} \\
{$[3,2,1,4,2]$} & {$[2,1,3,4,2]$} & {$[2,3,4,2]$} \\
{$[2,1,4,2]$} & {$[1,3,4,2]$} & {$[1,2,3,4,2]$} \\
\hline
\end{tabular}

\section{$\mathcal{P}(4,3)$}

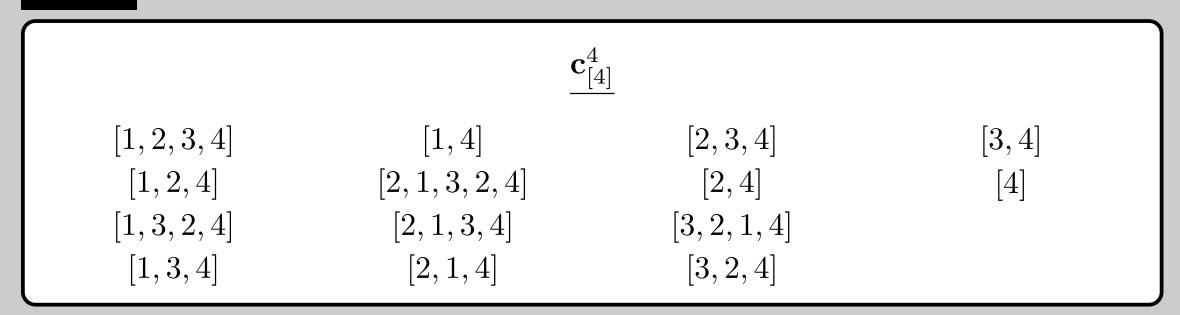

\section{$\mathcal{P}(4,4)$}

\begin{tabular}{|c|c|c|c|}
\hline \multicolumn{4}{|c|}{$\mathbf{c}_{[\text {[ ] }}^{4}$} \\
\hline$[1,2,3]$ & {$[1]$} & {$[2,3]$} & [3] \\
\hline$[1,2]$ & {$[2,1,3,2]$} & {$[2]$} & [ ] \\
\hline$[1,3,2]$ & {$[2,1,3]$} & {$[3,2,1]$} & \\
\hline$[1,3]$ & {$[2,1]$} & {$[3,2]$} & \\
\hline
\end{tabular}

Table 2.1: The packets of $D_{4}$ 


$\begin{array}{ccccccccc}1 & & & & & & & & \\ 1 & 1 & & & & & & \\ 1 & 1 & 1 & & & & & & \\ 1 & 1 & 1 & 1 & & & & & \\ 3 & 2 & 1 & 1 & 1 & & & & \\ 7 & 4 & 2 & 1 & 1 & 1 & & & \\ 15 & 8 & 4 & 2 & 1 & 1 & 1 & & \\ 31 & 16 & 8 & 4 & 2 & 1 & 1 & 1 & \\ \vdots & \vdots & \vdots & \vdots & \vdots & \vdots & \vdots & \vdots & \ddots\end{array}$

Table 2.2: Triangle of Packet Sizes

It can be built recursively: set the first entry $C(0,0)=1$, and then each subsequent entry is the sum of the entry above it and the entry to the left. All entries outside of the range $0 \leq k \leq n$ are considered to be 0 .

Table 2.3: Catalan's Triangle

More precisely, for $n \geq 0$ and $0 \leq k \leq n$, we define

$$
C(n, k)=\left\{\begin{array}{cl}
1 & \text { if } n=0 ; \\
C(n, k-1)+C(n-1, k) & \text { if } 0<k<n ; \\
C(n-1,0) & \text { if } k=0 ; \\
C(n, n-1) & \text { if } k=n .
\end{array}\right.
$$

The closed form for entries in this triangle is well known [OEI]. For $n \geq 0$ and $0 \leq k \leq n$, we have

$$
C(n, k)=\frac{(n+k) !(n-k+1)}{k !(n+1) !}
$$


One can also see that

$$
C_{n}=C(n, n-1)=C(n, n),
$$

where $C_{n}$ is the $n^{\text {th }}$ Catalan number.

Lemma 2.11. For $n \geq 4$, each collection in the packets $\mathcal{P}(n, n-1)$ and $\mathcal{P}(n, n)$ contains $C_{n}$ homogeneous words in $\mathcal{W}_{n}$.

Proof. Recall that each of these packets contains only one collection: $\mathcal{P}(n, n-$ $1)=\left\{\mathbf{c}_{[n]}^{n}\right\}$ and $\mathcal{P}(n, n)=\left\{\mathbf{c}_{[]}^{n}\right\}$. Since neither of the suffixes labeling these packets contains any of the letters $1,2, \ldots, n-1$, there are no restrictions on the homogeneous prefixes that can be included. Therefore, any homogeneous word of type $A_{n-1}$ can be a prefix. Since a fully commutative element is represented by a unique homogeneous word in canonical reduced form, there are exactly $C_{n}$ of homogeneous words of type $A_{n-1}$ by Proposition 1.7.

The previous lemma shows that the diagonal and subdiagonal of Catalan's triangle do in fact count the sizes of collections in the corresponding packets. We now give the main theorem of this section, which says that entries in the rest of Catalan's triangle also count the sizes of collections, naturally extending Lemma 2.11. This theorem allows us to classify the homogeneous representations.

Theorem 2.12. Assume that $n \geq 4$ and $0 \leq k \leq n$. Then any collection in the packet $\mathcal{P}(n, k)$ contains exactly $C(n, k)$ elements.

Proof. Note that Lemma 2.11 proves the case when $k=n-1$ or $n$. We next consider the packet $\mathcal{P}(n, 0)$, which consists of the collections labeled by the prefixes $s_{n 1} s_{n-1 j_{2}} s_{n j_{3}} s_{n-1 j_{4}} \cdots s_{n-1+[\ell]_{2} j_{\ell}}(\ell \geq 2)$. By Lemma 2.5, it is enough to consider the collection c labeled by $s_{n 1} s_{n-1}$. We claim that no prefix is possible except the empty word [ ]. Indeed, if a word $\boldsymbol{w}$ in $\mathbf{c}$ contains a non-empty prefix $\boldsymbol{w}_{0}$ ending with the letter $r$ for $1 \leq r \leq n-1$, then the word $\boldsymbol{w}$ contains, as a right factor, $[r, n, n-2, \ldots, 1, n-1]$ and we immediately reach a contradiction to homogeneity. Thus the collection c, and hence every collection in $\mathcal{P}(n, 0)$, contains only one element, the suffix itself. Since $C(n, 0)=1$, we are done with this case.

We see in Table 2.1 that any collection in the packet $\mathcal{P}(4, k)$ contains exactly $C(4, k)$ elements. Thus the assertion of the theorem is true for $n=4$. Recall the recursive definition of $C(n, k)$ : for $1 \leq k \leq n-1$,

$$
C(n, k)=C(n, k-1)+C(n-1, k) .
$$


We will prove that the collection sizes of the packets satisfy the relation given in equation (2.5). Since we already checked the base cases $(k=0, n=4)$, the theorem will be established by induction. Further, by Corollary 2.8, it will be enough to check that the relation holds for a single collection in each of the packets.

Now assume that $n>4$ and $1 \leq k \leq n-2$. We define

$$
\begin{aligned}
& \boldsymbol{w}_{1}=s_{n k} s_{n-1}=[n, n-2, \ldots, k, n-1], \quad \boldsymbol{w}_{2}=s_{n k}=[n, n-2, \ldots, k], \\
& \boldsymbol{w}_{3}= \begin{cases}{[n-1, n-3, \ldots, k]} & \text { if } k<n-2, \\
{[n-1]} & \text { if } k=n-2 .\end{cases}
\end{aligned}
$$

Then $\mathbf{c}_{\boldsymbol{w}_{1}}^{n} \in \mathcal{P}(n, k-1), \mathbf{c}_{\boldsymbol{w}_{2}}^{n} \in \mathcal{P}(n, k)$ and $\mathbf{c}_{\boldsymbol{w}_{3}}^{n-1} \in \mathcal{P}(n-1, k)$. We will give an explicit bijection from $\mathbf{c}_{\boldsymbol{w}_{1}}^{n} \cup \mathbf{c}_{\boldsymbol{w}_{3}}^{n-1}$ to $\mathbf{c}_{\boldsymbol{w}_{2}}^{n}$. Since $\mathbf{c}_{\boldsymbol{w}_{1}}^{n} \cap \mathbf{c}_{\boldsymbol{w}_{3}}^{n-1}=\emptyset$ as sets of formal words, we will have

$$
\left|\mathbf{c}_{\boldsymbol{w}_{1}}^{n}\right|+\left|\mathbf{c}_{\boldsymbol{w}_{3}}^{n-1}\right|=\left|\mathbf{c}_{\boldsymbol{w}_{2}}^{n}\right|,
$$

and the proof will be completed by induction since the collection sizes satisfy the same recursive relation as (2.5).

Define the map $\varphi_{1}: \mathbf{c}_{\boldsymbol{w}_{1}}^{n} \rightarrow \mathbf{c}_{\boldsymbol{w}_{2}}^{n}$ by

$$
\varphi_{1}\left(\boldsymbol{w}_{0} \boldsymbol{w}_{1}\right)=\varphi\left(\boldsymbol{w}_{0}[n, n-2, \ldots, k, n-1]\right)=\boldsymbol{w}_{0} \boldsymbol{w}_{2}=\boldsymbol{w}_{0}[n, n-2, \ldots, k] .
$$

Clearly, removing the last letter of the suffix will not affect the homogeneity of a word, and hence $\varphi_{1}$ maps $\mathbf{c}_{\boldsymbol{w}_{1}}^{n}$ into $\mathbf{c}_{\boldsymbol{w}_{2}}^{n}$. Similarly, we define the map $\varphi_{2}: \mathbf{c}_{\boldsymbol{w}_{3}}^{n-1} \rightarrow \mathbf{c}_{\boldsymbol{w}_{2}}^{n}$ to be

$$
\varphi_{2}\left(\boldsymbol{w}_{0} \boldsymbol{w}_{3}\right)=\boldsymbol{w}_{0} s_{n-1} \boldsymbol{w}_{2}=\boldsymbol{w}_{0} s_{n-1}[n, n-2, n-3, \ldots, k] .
$$

To see that the image is homogeneous, we first consider the case when $\boldsymbol{w}_{0}$ contains the letter $n-2$. In such a case, there are two neighbors, $n-1$ and $n$, between two occurrences of $n-2$. Now consider the other case when $\boldsymbol{w}_{0}$ contains $i<n-2$. In this case, there is a neighbor to the right of $i$ in $\boldsymbol{w}_{0}$ by homogeneity of $\boldsymbol{w}_{0} \boldsymbol{w}_{3}$ and also an $i+1$ in the suffix to the left of $i$. Hence, $\varphi_{2}$ maps $\mathbf{c}_{\boldsymbol{w}_{3}}^{n-1}$ into $\mathbf{c}_{\boldsymbol{w}_{2}}^{n}$. Note that the images of $\varphi_{1}$ and $\varphi_{2}$ are disjoint: the words in the image of $\varphi_{2}$ all have prefixes that end with the letter $n-1$, while none of the words in the image of $\varphi_{1}$ do since $\boldsymbol{w}_{0}$ cannot end with $n-1$ due to the homogeneity condition on $\boldsymbol{w}_{0} \boldsymbol{w}_{1}$.

Finally, we define the map $\varphi: \mathbf{c}_{\boldsymbol{w}_{1}}^{n} \cup \mathbf{c}_{\boldsymbol{w}_{3}}^{n-1} \rightarrow \mathbf{c}_{\boldsymbol{w}_{2}}^{n}$ to be the combination of $\varphi_{1}$ and $\varphi_{2}$, i.e. the restriction of $\varphi$ to $\mathbf{c}_{\boldsymbol{w}_{1}}^{n}$ is defined to be $\varphi_{1}$ and the 
restriction of $\varphi$ to $\mathbf{c}_{\boldsymbol{w}_{3}}^{n-1}$ is defined to be $\varphi_{2}$. Figure 2.1 shows an example of the maps $\varphi_{1}$ and $\varphi_{2}$ in the case of $n=5$ and $k=2$.

Now we define the map $\rho: \mathbf{c}_{\boldsymbol{w}_{2}}^{n} \rightarrow \mathbf{c}_{\boldsymbol{w}_{1}}^{n} \cup \mathbf{c}_{\boldsymbol{w}_{3}}^{n-1}$ to be given by the rule:

$$
\begin{aligned}
\rho\left(\boldsymbol{w}_{0} \boldsymbol{w}_{2}\right) & =\rho\left(\boldsymbol{w}_{0}[n, n-2, \ldots, k]\right) \\
& = \begin{cases}\boldsymbol{w}_{0}[n-3, \ldots, k] \in \mathbf{c}_{\boldsymbol{w}_{3}}^{n-1} & \text { if } \boldsymbol{w}_{0} \text { ends with } n-1, \\
\boldsymbol{w}_{0} \boldsymbol{w}_{1} \in \mathbf{c}_{\boldsymbol{w}_{1}}^{n} & \text { otherwise. }\end{cases}
\end{aligned}
$$

In the case where $\boldsymbol{w}_{0}$ ends with $n-1$, we first check homogeneity with the letter $n-3$ in $\boldsymbol{w}_{0}$. In passing to $D_{n-1}$, the letters $n-1$ and $n-3$ become neighbors and homogeneity follows because there is another neighbor of $n-3$ further to the right in $\boldsymbol{w}_{0}$. Now we check homogeneity when $\boldsymbol{w}_{0}$ contains $i<n-3$. Note that there is a neighbor to the right of $i$ in $\boldsymbol{w}_{0}$ by homogeneity of $\boldsymbol{w}_{0} \boldsymbol{w}_{2}$ and also an $i+1$ in the suffix to the left of $i$. In the case where $\boldsymbol{w}_{0}$ does not end with $n-1$, it is still possible that $\boldsymbol{w}_{0}$ contains $n-1$, but since it would be part of a descending sequence at the end of the prefix, there will always be two instances of the neighbor $n-2$ between the two occurrences of $n-1$. Thus the map $\rho$ is well defined.

Now one can check that $\rho$ is the two-sided inverse of $\varphi$. In particular, if we restrict $\rho$ to the words whose prefixes end with $n-1$, then we obtain the inverse for $\varphi_{2}$, while if we restrict to the prefixes not ending in $n-1$, we have the inverse for $\varphi_{1}$. This establishes (2.6) and completes the proof.

We obtain immediate consequences for homogeneous representations of $K L R$ algebras.

Corollary 2.13. Assume that $R=\bigoplus_{\alpha \in Q_{+}} R_{\alpha}$ is the type- $D_{n}$ KLR algebra.

(1) The set of irreducible homogeneous representations of $R$ is decomposed into packets and collections according to the decomposition of the set of fully commutative elements (or of the set of homogeneous words in $\left.\mathcal{W}_{n}\right)$.

(2) Each entry in Catalan's triangle counts the number of homogeneous representations of $R$ in the same collection.

Corollary 2.14. For $n \geq 4$, we obtain the identity:

$$
\sum_{k=0}^{n} C(n, k)|\mathcal{P}(n, k)|=\frac{n+3}{2} C_{n}-1
$$

Proof. The identity follows from Proposition 1.7 and Theorem 2.12. 


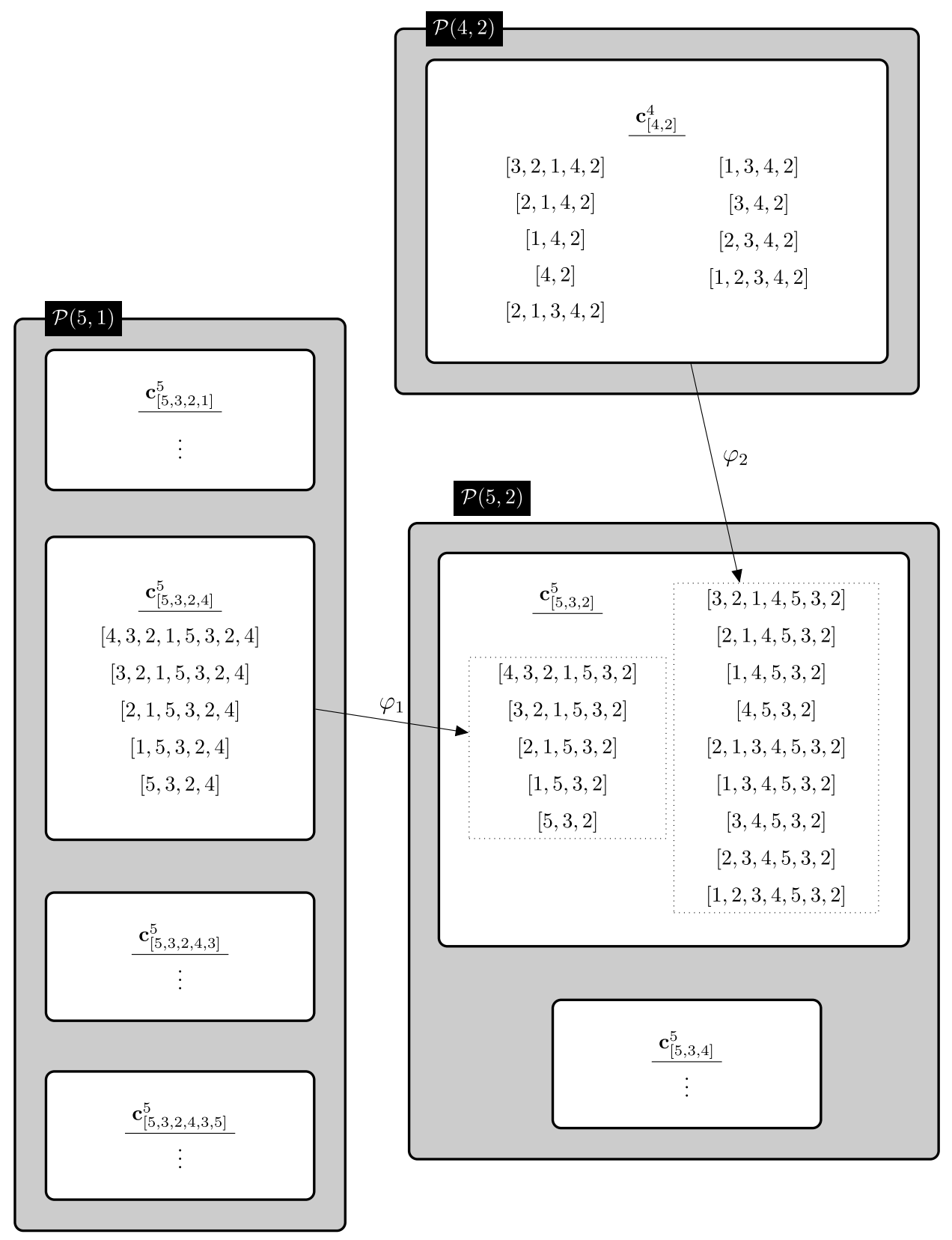

Figure 2.1: The maps $\varphi_{1}$ and $\varphi_{2}$ into the packet $\mathcal{P}(5,2)$. 
Remark 2.15. As pointed out in the introduction, the identity (2.7) suggests that there might be a representation theoretic construction, in which $C(n, k)$ would correspond to the dimension of a representation and $|\mathcal{P}(n, k)|$ to its multiplicity. Also recall that the right-hand side of (2.7) is equal to the dimension of the Temperley-Lieb algebra of type $D_{n}$.

\section{References}

[BS01] Leonid A. Bokut and Long-Sheng Shiao, Gröbner-Shirshov bases for Coxeter groups, Comm. Algebra 29 (2001), no. 9, 4305-4319, Special issue dedicated to Alexei Ivanovich Kostrikin. MR1857281

[Fan95] C. Kenneth Fan, A Hecke algebra quotient and properties of commutative elements of a Weyl group, M.I.T., 1995, Ph.D. thesis. MR2716583

[Fan96] C. Kenneth Fan, A Hecke Algebra quotient and some combinatorial applications, J. Algebraic Combin. 5 (1996), no. 3, 175-189. MR1394304

[Fan97] C. Kenneth Fan, Structure of a Hecke algebra quotient, J. Amer. Math. Soc. (1997), no. 1, 139-167. MR1396894

[FG99] C. Kenneth Fan and R. M. Green, On the affine Temperley-Lieb algebras, J. London Math. Soc. (2) 60 (1999), no. 2, 366-380. MR1724798

[GL01] R. M. Green and J. Losonczy, Fully commutative KazhdanLusztig cells, Ann. Inst. Fourier (Grenoble) 51 (2001), no. 4, 1025-1045. MR1849213

[Gra95] J. J. Graham, Modular Representations of Hecke Algebras and Related Algebras, University of Sydney, 1995, Ph.D. thesis.

[HMM12] David Hill, George Melvin, and Damien Mondragon, Representations of quiver Hecke algebras via Lyndon bases, J. Pure Appl. Algebra 216 (2012), no. 5, 1052-1079. MR2875327

[Jon87] Vaughan F. R. Jones, Hecke algebra representations of braid groups and link polynomials, Ann. of Math. (2) 126 (1987), no. 2, 335-388. MR0908150

[KL09] Mikhail Khovanov and Aaron D. Lauda, A diagrammatic approach to categorification of quantum groups I, Represent. Theory 13 (2009), no. 09, 309-347. MR2525917

[KR10] Alexander Kleshchev and Arun Ram, Homogeneous representations of Khovanov-Lauda Algebras, J. Eur. Math. Soc. 12 (2010), no. 5, 1293-1306. MR2677617 
[KR11] Alexander Kleshchev and Arun Ram, Representations of Khovanov-Lauda-Rouquier algebras and combinatorics of Lyndon words, Math. Ann. 349 (2011), no. 4, 943-975. MR2777040

[Lec04] Bernard Leclerc, Dual canonical bases, quantum shuffles and qcharacters, Math. Z. 246 (2004), no. 4, 691-732. MR2045836

[LS13] Tobias Lejczyk and Catharina Stroppel, A graphical description of $\left(D_{n}, A_{n-1}\right)$ Kazhdan-Lusztig polynomials, Glasg. Math. J. 55 (2013), no. 2, 313-340. MR3040865

[Lus11] George Lusztig, Introduction to quantum groups, Springer, 2011. MR2759715

[OEI] Sequence A009766, http://oeis.org/A009766, The On-Line Encyclopedia of Integer Sequences, published electronically at http://oeis.org.

[Rou08] Raphaël Rouquier, 2-Kac-Moody algebras, arXiv:0812.5023 [math.RT].

[Ste96] John R. Stembridge, On the fully commutative elements of Coxeter groups, J. Algebraic Combin. 7 (1996), 353-385. MR1406459

[Ste97] John R. Stembridge, Some combinatorial aspects of reduced words in finite Coxeter groups, Trans. Amer. Math. Soc. (1997), no. 4, 1285-1332. MR1389789

[Ste98] John R. Stembridge, The enumeration of fully commutative elements of Coxeter groups, J. Algebraic Combin. 7 (1998), no. 3, 291-320. MR1616016

Gabriel FeinberG

Department of Mathematics and Statistics

HAVERFord COLLEGE

HAVERFORD, PA 19041

U.S.A.

E-mail address: gfeinberg@haverford.edu

KyU-Hwan LeE

Department of Mathematics

UNIVERSITY OF CONNECTICUT

StORRs, CT 06269

U.S.A.

E-mail address: khlee@math.uconn.edu

Received 18 ApriL 2014 